\title{
Validação das intervenções e atividades de enfermagem para pacientes com Insuficiência Cardíaca
}

\section{Larissa C Figueiredo*, Erika C M Duran.}

\section{Resumo}

O presente estudo objetivou validar as intervenções e atividades de enfermagem para pacientes hospitalizados com Insuficiência Cardíaca e diagnóstico de enfermagem de Intolerância à Atividade. Tratou de um estudo metodológico, transversal, de validação de intervenções a partir da terminologia de Classificações de Intervenções de Enfermagem. $\mathrm{Na}$ primeira etapa foi realizada uma revisão integrativa da literatura para a identificação de intervenções e atividades existentes na referida classificação e a segunda etapa consistiu na analise do conteúdo por especialistas. Obteve-se seis artigos na primeira etapa que apresentaram dez intervenções de enfermagem e suas atividades, e foi validado pelos especialistas nove intervenções e suas atividades. Esse estudo apresentou subsídios que pode colaborar para a prática clínica do enfermeiro na prestação de um cuidado qualificado para a população estudada.

\section{Palavras-chave: Processos de Enfermagem, Cuidados de Enfermagem, Enfermagem Cardiovascular.}

\section{Introdução}

A insuficiência cardíaca é uma doença sistêmica em que se há o comprometimento do bombeamento $e$ enchimento cardíaco, resultando em um suprimento sanguíneo inadequado para atender as demandas metabólicas do organismo, causando grandes limitações para o paciente ${ }^{1}$. Nesse contexto, o enfermeiro realiza a avaliação do paciente, levando em consideração quais são as necessidades básicas do individuo e como ele responde a doença ${ }^{2}$. $\mathrm{Na}$ insuficiência cardíaca, verificouse que o diagnóstico Intolerância à atividade tem grande incidência nos pacientes hospitalizados ${ }^{3}$. Assim, o objetivo deste trabalho foi de validar Intervenções e Atividades de Enfermagem propostas na Classificação de Intervenções de Enfermagem $(\mathrm{NIC})^{4}$ para o Diagnóstico de Enfermagem de Intolerância a Atividade para pacientes com Insuficiência Cardíaca que estão hospitalizados.

\section{Resultados e Discussão}

Tratou de um estudo metodológico, transversal, de validação de intervenções a partir da terminologia Classificações de Intervenções de Enfermagem (NIC). A primeira etapa contou com uma revisão integrativa da literatura, no qual a pesquisa foi realizada nas bases de dados COCHRANE, SCOPUS, MEDLINE, LILACS e CINAHL, utilizando os descritores "Insuficiência Cardíaca", "Cuidados de Enfermagem" e "Processos de Enfermagem".

A partir da revisão obtiveram-se seis artigos que apresentaram 10 intervenções de enfermagem diferentes para a população com Insuficiência Cardíaca, mas não especifico para o diagnóstico de enfermagem de Intolerância à atividade. Sendo assim, dentre as intervenções que os artigos apresentaram, foram selecionadas as que estavam nas ligações entre as intervenções da NIC e os diagnósticos da NANDA Internacional para o diagnóstico de enfermagem trabalhado e foram selecionadas atividades de enfermagem que se mostraram relevantes para a população estudada.

A segunda etapa constituiu em análise do conteúdo das intervenções e atividades por especialistas que foram selecionados com base nos currículos disponíveis na Plataforma Lattes e por indicações de enfermeiros previamente selecionados. Cada intervenção de enfermagem foi avaliada segundo sua relação com a população e suas atividades foram avaliadas segundo a relevância, clareza e precisão, utilizando a escala Likert de cinco pontos: 1- nada característico; 2- muito pouco característico; 3- um pouco característico; 4consideravelmente característico; 5- muito característico. Foi aplicado o teste estatístico binomial para estabelecer se, proporcionalmente, o número de concordância entre os especialistas foi maior ou igual ao valor $85 \%$ de concordância entre especialistas, e assim foram validadas nove intervenções de enfermagem, e algumas de suas atividades, sendo elas: Assistência no autocuidado, Controle da Nutrição, Controle de arritmias, Controle de energia, Controle de Medicamentos, Cuidados Cardíacos, Ensino: Exercício Prescrito, Promoção do Envolvimento Familiar e Terapia com Exercício: Deambulação ${ }^{4}$.

\section{Conclusões}

A partir dessa pesquisa foi possível validar nove intervenções e 84 atividades de enfermagem. O paciente com Insuficiência Cardíaca necessita de uma assistência que abranja aspectos fisiológicos, psicológicos e sociais. Assim, esse estudo levantou cuidados de enfermagem mais específicos na área prática clinica, fornecendo subsídios para sua qualidade e realizando um refinamento das terminologias utilizadas.

\section{Agradecimentos}

A presente iniciação científica teve como instituição de fomento o Conselho Nacional de Desenvolvimento Científico e Tecnológico (CNPq).

\footnotetext{
1 Tratado de enfermagem médico-cirúrgica: avaliação e assistências dos problemas clínicos/ [Sharon L. Lewis et al.]; [Tradução Maiza Ritimy Ide] . Rio de Janeiro: Elsevier, 2013. 1802 p.

${ }^{2}$ Thoroddsen A, Ehnfors M, Nurs D, Nurs A. Nursing Specialty Knowledge as Expressed by Standardized Nursing Languages. International Journal of Nursing Terminologies and Classifications. 2010 April; 21(Issue 2): 69-79.

${ }^{3}$ Pereira JMV, Flores PVP, Figueiredo LS, Arruda CS, Cassiano KM, Vieira GCA et al. Diagnósticos de enfermagem em pacientes com insuficiência cardíaca hospitalizados: estudo longitudinal. Rev. esc. enferm. USP. 2016: 929-936.

${ }^{4}$ Bulechek GM, Butcher HK, Dochterman JM, Wagner CM. Classificação das intervenções de enfermagem (NIC). Rio de Janeiro: Elsevier; 6 ed. 2016.
} 